

\title{
Variación espacio-temporal en la actividad relativa de murciélagos insectívoros aéreos en la Reserva de la Biosfera Sierra de Huautla, Morelos, México
}

\section{Spatial-temporal variation in the relative activity of aerial insectivore bats in the Sierra de Huautla Biosphere Reserve, Morelos, Mexico}

\author{
Carmen Lorena Orozco-Lugo ${ }^{{ }^{*}}$, Antonio Guillen-Servent ${ }^{2}$, \\ David Valenzuela-Gálvann ${ }^{1}$, Héctor T. Arita ${ }^{3}$ y Nestor A. Mariano-Bonigo ${ }^{1,4}$.
}

\section{Resumen}

La dinámica ecológica de los murciélagos insectívoros aéreos es poco conocida, especialmente para las selvas secas en América, un tipo de vegetación en riesgo. Exploramos el efecto de la perturbación de la vegetación, la estacionalidad climática y del microhábitat en la actividad relativa en este ensamble de quirópteros. Trabajamos en tres áreas de la REBIOSH, seleccionando en cada una dos sitios contrastantes en su grado de perturbación (evaluada con base en la cobertura forestal y de uso agroforestal); en cada sitio se establecieron dos transectos, uno en ladera, otro en cañada. En ellos se realizaron grabaciones ultrasónicas durante el primer pico de actividad de los murciélagos durante septiembre - octubre de 2001 y abril - mayo de 2002 para determinar índices de actividad por especie o fonotipo. El índice de actividad para Balantiopterix plicata y para el fonotipo vespertiliónido difirió significativamente entre temporadas; probablemente por movimientos altitudinales o regionales con fines reproductivos, o por la variación estacional en la abundancia de recursos. Los forrajeadores de borde tuvieron mayor actividad en los sitios perturbados; los molósidos y Pteronotus parnellii no tuvieron diferencias significativas en ninguno

\footnotetext{
${ }^{1}$ Centro de Investigación en Biodiversidad y Conservación - UAEM. Av. Universidad 1001, Chamilpa, Cuernavaca 62209, Morelos. México.

${ }^{2}$ Departamento de Biodiversidad y Ecología Animal. Instituto de Ecología, A. C., Km 2.5 Antigua Carretera a Coatepec 351. Congregación El Haya, Xalapa 91070, Veracruz, México.

${ }^{3}$ Laboratorio de Macroecología. Centro de Investigaciones en Ecosistemas, UNAM. Apartado Postal 27-3. Morelia 58089, Michoacán. México.

${ }^{4}$ Instituto de Ambiente de Montaña y Regiones Áridas (IAMRA). Universidad Nacional de Chilecito. 9 de julio 22. Chilecito. La Rioja. Argentina.C.P.: F5360CKB

*Corresponding autor: zotz@uaem.mx
} 
de los factores, lo cual se esperaba para los molósidos, pero en el caso de P. parnellii sugiere que es una especie capaz de utilizar sitios con cierto nivel de perturbación y no solo hábitats estructuralmente complejos. Por último, P. davyi, P. personatus, Mormoops megalophylla, B. plicata y el fonotipo vespertiliónido fueron significativamente más activos en cañadas que en laderas; estos datos apoyan la idea de que el ensamble prefiere las cañadas para llevar a cabo sus actividades, sin importar el grado de perturbación de los sitios. La conservación de las cañadas debería enfatizarse entre las acciones de manejo de esta ANP y proponemos que sean consideradas sitios para monitoreo a largo plazo en ambientes secos altamente estacionales.

Palabras clave: Selva baja. Cañadas. Vegetación riparia. Detección ultrasónica.

\section{Abstract}

The ecological dynamics of aerial insectivore bats is poorly known especially for the tropical dry forests (TDF) of America, an endangered habitat. This study explored the effect of tropical dry forest disturbance, its climatic seasonality, and the microhabitat type in the relative activity of aerial insectivore bats. It was conducted at three areas within the Sierra de Huautla Biosphere Reserve in the state of Morelos, México; two sites with contrasting disturbance level (based on forest and agroforestry use cover) were chosen from each area. Two sampling transects were established in each site, one on a hillside and the other one on a river creek, obtaining ultrasonic recordings of bat activity during the first part of the night, on sampling nights from September-October 2001 and April-May 2002 to determine activity index by species. The activity index for B. plicata and for the vespertilionid phonotype differed significantly between climatic seasons, possibly in relation to altitudinal or regional movements linked to reproduction or to the seasonal variation on resources. Forest edge foragers showed higher activity on disturbed sites. For molossids and for P. parnellii no significant differences were found between the considered factors, which in turns suggests that P. parnellii is a species able to use sites with certain disturbance level and not only structurally complex sites. Finally, B. plicata, P. davyi, P. personatus, M. megalophylla and species within the vespertilionid phonotype were significantly more active at river creeks than at hillsides. Our data supports the idea that the studied bat ensemble requires this microhabitat 
for its activities, independently of the level of conservation of the surrounding vegetation. River creeks should be considered a management priority on this natural protected area and should be sites for long term monitoring of aerial insectivore bats at highly seasonal sites of TDF.

Key words: Tropical dry forest. River creeks. Riparian vegetation. Ultrasound detectors.

\section{Introducción}

Las selvas secas de América Latina están sujetas a una fuerte presión y perdida de hábitat, se estima que solo el 27\% de ellas, con relación a la distribución potencial o histórica se encuentra intacta, otro $27 \%$ ha sido alterada y el 46 \% ha sido degradada o convertida (Trejo y Dirzo, 2000). Además de esta problemática, es evidente que la generación de conocimiento científico sobre los patrones ecológicos de estas selvas ha sido limitado, y es solo en las últimas dos décadas que ha cobrado preponderancia (Sánchez-Azofeifa et al., 2005).

En el caso particular del orden Chiroptera, Mickleburgh et al. (2002) hacen énfasis en que el conocimiento de la diversidad de este grupo taxonómico en las selvas secas del mundo es escaso y prioritario en este aspecto por dos motivos: la elevada tasa de desaparición de este tipo de vegetación y el elevado número de endemismos de murciélagos que ahí se encuentran. Para México la tasa de desaparición de las selvas secas está estimada en 1.4\% anual para algunas regiones del país (Trejo y Dirzo, 2000); respecto a los murciélagos, siete de las 15 especies endémicas de México tienen distribución exclusiva en este tipo de vegetación, cinco de ellas son insectívoras y dos nectarívoras (Ceballos, 1995). Por lo anterior, identificar patrones ecológicos sobre la diversidad y uso de hábitat de los murciélagos en las selvas secas, aporta elementos que contribuyen al manejo de las mismas.

En el caso particular de los murciélagos insectívoros aéreos, la información sobre su presencia y abundancia en las selvas secas del neotrópico mexicano está poco documentada, debido entre otras cosas a que no son consistentemente capturados en redes (Kalko et al., 1996; O’Farrell y Gannon, 1999).

Datos generados en otras regiones tropicales, indican que en áreas deforestadas de la Guayana francesa (Brosset et al., 1996), los vespertiliónidos y molósidos se mantienen activos mientras otras 
especies prácticamente desaparecen. Reis y Muller (1995) encuentran en el bosque amazónico, que las capturas de murciélagos insectívoros con redes de niebla son mayores en los fragmentos de bosque más grandes (680 ha) y casi nulas en los fragmentos pequeños ( 60 ha.), en este caso la mayor captura está dada principalmente por una especie forrajeadora de sustrato; otros trabajos reportan un incremento de molósidos y disminución de insectívoros de sustrato en bosques explotados (Ochoa, 2000). Por último, Estrada et al. (2004) registran la actividad general de murciélagos insectívoros mediante ultrasonidos (sin tomar en cuenta especies o fonotipos), en fragmentos de selva perennifolia en la región de Los Tuxtlas, Veracruz, encontrando diferencias en los niveles de actividad entre los distintos tipos de vegetación, siendo mayor la actividad en los fragmentos de selva y la vegetación riparia, y casi nula en los pastizales.

El impacto de la perturbación del hábitat en la riqueza, así como en la abundancia y patrones de actividad de los murciélagos insectívoros no es claro (Fenton, 1997). La configuración de un paisaje formado por distintos tipos de vegetación, que incluya agricultura orgánica o no intensiva, así como cultivos de sombra en bosques en diferentes estados sucesionales, parece no tener un efecto negativo sobre las poblaciones de murciélagos insectívoros y de los quirópteros en general (Estrada et al., 2004; Patriquin y Barclay, 2003; Pineda et al., 2005; Wickramasinghe et al., 2003); es importante resaltar que fragmentos de bosque conservado o de viejo crecimiento dentro de un mosaico de vegetación, proveen de microhábitats específicos de forrajeo y refugios para los murciélagos, por lo que contribuyen al mantenimiento de la diversidad (Gorresen y Willing, 2004; Zubaid, 1993).

La respuesta de las especies a la perturbación, al parecer, está directamente relacionada con el tamaño corporal de los murciélagos -las especies de mayor tamaño toleran mejor la perturbación (Patriquin y Barclay, 2003; Webala et al., 2004)- la especialización en la dieta (Stoner et al., 2002) y las limitaciones y compromisos impuestos por las características del sistema de ecolocación y la morfología alar (Aldridge y Rautenbach, 1987; Glendell y Vaughan, 2002; Neuweiler, 1989; Patriquin y Barclay, 2003; Schnitzler y Kalko, 1998).

Por otra parte, varios estudios han sugerido que la distribución de murciélagos en diferentes hábitats, así como sus niveles de actividad, se pueden explicar por factores extrínsecos como la disponibilidad de insectos (Bell, 1980; Brigham et al., 1992; Jong y Ahlén, 1991; Jong, 1994), la presencia de cuerpos de agua (ríos, arroyos y lagos) y la disponibilidad de refugios (Glendell 
y Vaughan, 2002; Korine y Pinshow, 2004; Meyer et al., 2004; Rautenbach et al., 1996; Seidman y Zabel, 2001; Wickramasinghe et al., 2003).

Dada la escasa información de que disponemos sobre la diversidad y dinámicas espacio temporales de los murciélagos insectívoros aéreos en las selvas secas de México, el objetivo de este trabajo fue explorar el efecto de la perturbación de la vegetación, la estacionalidad climática (seca vs lluvias) y la influencia del microhábitat (laderas vs cañadas) en la actividad relativa de este ensamble; este ambiente, al ser altamente estacional, nos permite poner a prueba la importancia de las corrientes de agua y de la vegetación asociada a ella, ya que se ha reportado en la literatura como un microhábitat fundamental en la presencia de los murciélagos (Avila-Cabadilla et al., 2012; Glendell y Vaughan, 2002; Korine y Pinshow, 2004; MacSwiney et al., 2007; Meyer et al., 2004; Rautenbach et al., 1996; Seidman y Zabel, 2001; Wickramasinghe et al., 2003).

Considerabamos que aquellas especies de murciélagos que forrajean alejadas de la vegetación (ejem. molósidos, algunos vespertiliónidos y Balantiopterix plicata (Peters), no se verían afectadas por la perturbación del hábitat. Las especies que forrajean en el borde de la vegetación o huecos de la misma, por ejemplo miembros de la familia Vespertilionidae (Myotis spp.) y algunas especies de la familia Mormoopidae: Pteronotus davyi (Gray), Pteronotus persontaus (Wagner), Mormoops megalophylla (Peters), podrían aumentar su actividad en las zonas perturbadas o de ladera. Por último de, Pteronotus parnellii (Gray), murciélago especializado en el forrajeo de espacios con alta complejidad estructural (Schnitzler y Kalko, 2001; Schnitzler et al., 2003), sería más activo en sitios no perturbados. Esperabamos que la actividad fuera mayor durante la estación de lluvias (debida quizá a la mayor abundancia de presas) que en la estación seca, y que en las cañadas hubiera significativamente más actividad que en las laderas, por mantener la humedad por más tiempo durante el año, siendo con esto, ambientes benignos, sobre todo en la temporada seca del año.

\section{Materiales y métodos}

Sitio de estudio

La Reserva de la Biosfera Sierra de Huautla (REBIOSH) se encuentra al sur del Estado de Morelos, México (Fig. 1); el tipo de vegetación predominante en la zona es la selva baja caducifolia, la precipitación promedio es de $900 \mathrm{~mm}$ anuales entre junio y octubre, con una larga temporada seca el resto del año. Las principales amenazas que se han identificado para la zona son la tala para venta de madera o leña, la cacería de subsistencia y la ganadería extensiva (Valenzuela et al., 2010). 


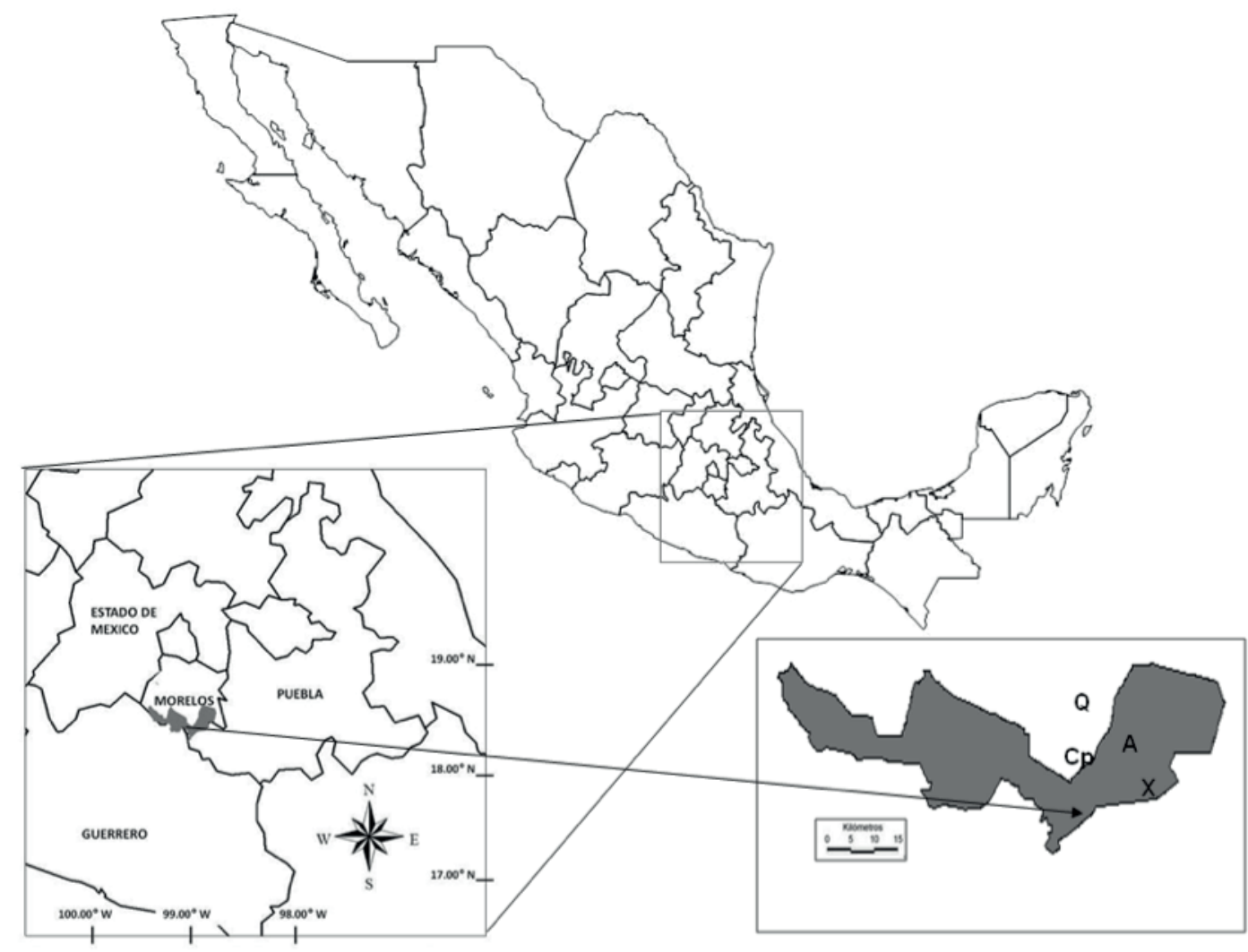

Fig. 1. Mapa de localización de la Reserva de la Biosfera Sierra de Huautla (REBIOSH). En el recuadro inferior derecho se muestra el contorno de la REBIOSH, las letras se refieren a los sitios de muestreo: Q (Quilamula), Cp (Cruz pintada), A (Ajuchitlán), X (Xantiopan).

\section{Los murciélagos insectívoros aéreos}

De las 41 especies de murciélagos que se han reportado para la zona, 26 se alimentan de insectos y pertenecen a seis familias (Orozco-Lugo et al., 2014; Rhodes, 2005; Sánchez-Hernández y Romero, 1995). De estas 26 especies, 24 son forrajeadores aéreos y es factible su registro grabando los llamados de ecolocalización con detectores de ultrasonido; Natalus mexicanus (Miller) sería la excepción, ya que tiene llamados de ecolocalización de baja intensidad (Rydell et al., 2002), por lo que seguramente no es detectado consistentemente en las grabaciones (Anexo 1).

La identificación de las especies por sus pulsos de ecolocalización se basó en descripciones previas para los llamados (Adams, 1989; Ibáñez et al., 1999; Ibáñez et al., 2002; Macias et al., 2006; Novick, 1962; O’Farrell y Miller, 1997; O’Farrell et al., 1999; Parsons y Jones, 2000; Ratcliffe et al., 2004; Rydell et al., 2002); lo anterior, junto con el análisis de grabaciones hechas en el sitio (OrozcoLugo et al., 2013), nos permiten, para el propósito de nuestros análisis, considerar la correcta 
identificación de cinco especies (Fig. 2): Balantiopterix plicata (BAL), Pteronotus parnellii (PAR), P. personatus (PER), P. davyi (DAV) y Mormops megalophylla (MOR); el resto fueron agrupadas en dos grupos o fonotipos, procedimiento recomendable cuando no se conocen detalladamente los sonidos de ecolocalización y que ha sido utilizado por otros autores (Hayes, 1997, 2000).

En nuestro caso, estos fonotipos se caracterizaron por la inclusión de especies de murciélagos que emiten pulsos de ecolocalización de frecuencia modulada (FM) o frecuencia constante (FC) en dos familias. La primera de ellas, Vespertilionidae (Fig. 2), que incluiría a las siguientes especies: Myotis californicus (Audubon y Bachman), Myotis fortidens (Miller y Allen), Myotis yumanensis (Allen), Myotis velifer (Allen), Lasiurus blossevilli (Lesson y Garnot), Lasiurus cinereus (Palisot de Beauvois), Lasiurus intermedius (Allen), Parastrellus hesperus (Allen), Rhogeessa alleni (Thomas) Rhogeessa gracilis (Miller) y Rhogeessa pároula (Allen); el segundo fonotipo es el de la familia Molossidae, con las siguientes especies: Eumops underwoodi (Goodwin), Eumops glaucinus (Wagner), Molossus sinaloae (Allen), Nyctinomops femorosaccus (Merriam), Nyctinomops macrotis (Gray) y Tadarida brasiliensis (Geoffroy).

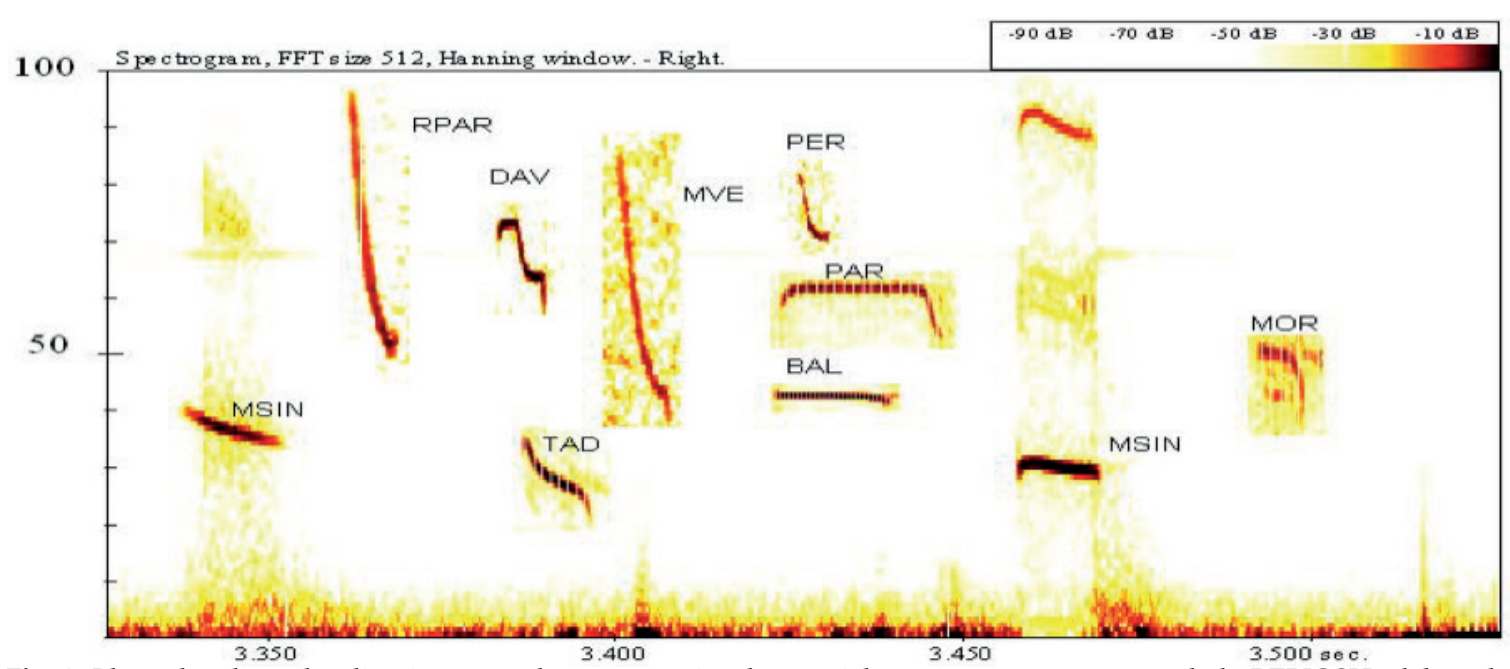

Fig. 2. Llamados de ecolocalización para algunas especies de murciélagos insectívoros aéreos de la REBIOSH, elaborados a partir del programa BatSound $\odot$. El eje de las abscisas (" $x$ ") corresponde a milisegundos y el de las ordenadas (" $y$ ") a KHz. $\mathrm{DAV}=$ Pteronotus davyi, $\mathrm{PER}=$ Pteronotus personatus, $\mathrm{PAR}=$ Pteronotus parnellii, $\mathrm{BAL}=$ Balantiopterix. plicata, $\mathrm{MOR}=$ Mormoops megalophylla; dentro del fonotipo "molósido: se incluyeron especies como: MSIN = Molossus sinaloae, en este caso se representan dos pulsos de la misma secuencia, esta alternancia de pulsos es característica del genero Molossus y TAD = Tadarida brasiliensis; dentro del fonotipo "vespertiliónido" se incluyen especies como: RPAR = Rhogeessa párvula y MVE = Myotis velifera.

\section{Censos de actividad}

Durante septiembre - octubre de 2001 y abril - mayo de 2002, se trabajó en tres áreas de la REBIOSH: Xantiopa $\left(\mathrm{X}, 18.44^{\circ} \mathrm{N}, 98.99^{\circ} \mathrm{O}\right)$, Ajuchitlán $\left(\mathrm{A}, 18.46^{\circ} \mathrm{N}, 98.99^{\circ} \mathrm{O}\right)$ y Cruz Pintada $\left(\mathrm{Cp}, 18.46^{\circ} \mathrm{N}\right.$, 
$\left.99.03^{\circ} \mathrm{O}\right)$; para el muestreo de junio - julio de 2002, el sitio de Cruz Pintada no pudo seguir siendo utilizado y se escogió el área de Quilamula $\left(\mathrm{Q}, 18.51^{\circ} \mathrm{N}, 99.01^{\circ} \mathrm{O}\right)$, para completar tres áreas de estudio en esta fecha. La distancia mínima entre cada sitio fue de $3 \mathrm{~km}$ y la máxima de 8.4. Para la elección de estas áreas se buscó tener sitios contrastantes en cuanto al grado de alteración de la vegetación natural, además se tomaron en cuenta aspectos logísticos que permitieran el traslado y trabajo en ellos de manera simultánea.

Dentro de estas áreas generales se seleccionaron dos sitios contrastantes en su grado de perturbación, inferida por el uso agroforestal, la estructura y cobertura vegetal, todo ello con base en datos de cobertura, determinada para cuadros de $1 \mathrm{~km}^{2}$ en el mapa de vegetación de la REBIOSH (Elaborado por el Laboratorio Interdisciplinario de Sistemas de Información Geográfica del Centro de Investigación en Biodiversidad y Conservación). Las características de los sitios perturbados (P) fueron: ganado constante y abundante, sotobosque escaso, presencia de pastos, estructura arbórea homogénea y poco densa, cobertura escasa y presencia de tierras de cultivo. Los sitios perturbados presentan árboles aislados, o árboles formando cercas vivas delimitando las tierras de cultivo. Los sitios no perturbados (NP) presentan: ganado poco abundante y no presente todo el tiempo, sotobosque abundante, pocos pastos o ausentes en el sitio, estructura arbórea heterogénea y densa, con cobertura continua.

Para los sitios NP se obtuvo el valor de diversidad de árboles promedio mediante el índice de Simpson, D = 25.36, mientras que en los sitios perturbados fue de D = 14.198 (Cadena-Salgado, 2003). Estos sitios se ubicaron en la misma microregión (ejem. misma cuenca), para homogeneizar el efecto de factores no evaluados, como la historia de uso del suelo. Para evaluar el efecto del microhábitat, en cada uno de estos sitios se establecieron dos transectos de $2 \mathrm{~km}$ de longitud, uno en ladera (L) y otro en cañada (C).

En los transectos de X, A y Cp se efectuaron censos de murciélagos durante dos noches en el periodo de septiembre-octubre 2001, lo que corresponde al final de la temporada de lluvia (lluvias tardías) y abril-mayo 2002, que corresponde a la temporada seca. Para el periodo de junio-julio de 2002, solo se pudo realizar una noche de muestreo para cada transecto en los sitios X, A y Q; este periodo corresponde al principio de la época de lluvias (lluvias tempranas). 


\section{Análisis estadístico}

Efecto de la perturbación de la vegetación, temporada y microhábitat

Para evaluar el efecto de la perturbación (P, NP), temporada (secas, lluvias tardías y lluvias tempranas) y microhábitat (L, C), sobre la actividad relativa de los murciélagos insectívoros, en cada noche de grabación se determinó la presencia/ausencia de cada especie o fonotipo en periodos de un minuto (Miller, 2001), posteriormente se sumó el total de minutos con presencia de la especie o fonotipo en cada noche y esto se consideró el índice de actividad. Para permitir la evaluación de la variación del índice de actividad entre los factores, fue necesario calcularlo sobre la misma ventana temporal de grabación en cada noche, ello debido a que no todas las grabaciones se pudieron iniciar con la puesta astronómica, por eso solo se consideraron 46 noches, todas con grabación efectiva entre los treinta y 114 minutos.

Esta información se analizó mediante un análisis multivariante de la varianza protegido con efecto de bloque (MANOVA), en el que microhábitat se anidó a condición de perturbación (NP y P). Cada sitio de muestreo fue considerado como una réplica para cada uno de los factores: perturbación, temporada y microhábitat.

\section{Resultados}

Con relación a la temporada, el índice de actividad promedio de Balantiopterix plicata difirió significativamente entre temporadas $y$ fue cerca de nueve veces mayor en secas que en cualquiera de las dos temporadas de lluvia, pero no difirió entre temporadas de lluvia (Tabla I). El índice de actividad promedio del fonotipo vespertiliónido (VESP) fue significativamente mayor (cerca de tres veces más) durante la temporada de lluvias tempranas que en las otras dos temporadas (Tabla I). Para el resto de las especies no se observan diferencias significativas con relación a la temporada.

Respecto al efecto de la perturbación, M. megalophylla y el fonotipo VESP presentaron índices de actividad promedio significativamente mayores en los sitios perturbados (cerca del doble en ambos casos, Tabla II); para el resto de las especies y el fonotipo molósido (MOL) no se detectaron diferencias de actividad relacionadas con la perturbación. En el caso particular de B. plicata la diferencia en el promedio del índice de actividad entre sitios perturbados y no perturbados arrojó 
un valor de significancia marginal (Tabla II). En relación al microhábitat (laderas vs cañadas), con excepción de P. parnellii y el fonotipo MOL, los índices de actividad promedio del resto de las especies fueron significativamente mayores en las cañadas que en las laderas (entre una y tres veces mayores; Tabla II).

Tabla I. Promedio de la actividad ( $\mathrm{min} /$ noche) por especie por temporada. La significancia de cada comparación se basa en los resultados del MANOVA, en donde * $=P<0.05$

\begin{tabular}{lccc}
\cline { 2 - 3 } & \multicolumn{2}{c}{ TEMPORADA } & \\
\hline ESPECIE & Lluvias tempranas & Seca & Lluvias tardías \\
Pteronotus parnellii & $10.08 \pm 2.54$ & $7.41 \pm 1.84$ & $9.53 \pm 1.34$ \\
Pteronotus davyi & $9.83 \pm 1.66$ & $8.00 \pm 1.21$ & $13.47 \pm 2.94$ \\
Pteronotus personatus & $2.91 \pm 1.24$ & $2.82 \pm 0.87$ & $1.93 \pm 0.75$ \\
Mormoops megalophylla & $6.08 \pm 1.79$ & $6.32 \pm 1.29$ & $7.47 \pm 2.62$ \\
Balantiopterix plicata & $2.83 \pm 1.03$ & $\mathbf{1 8 . 6 8} \pm \mathbf{1 . 5 0 *}$ & $1.87 \pm 1.11$ \\
Fonotipo VESP & $\mathbf{2 2} \pm \mathbf{2 . 7 6 ^ { * }}$ & $7.50 \pm 2.05$ & $5.00 \pm 1.57$ \\
Fonotipo MOL & $8.67 \pm 3.49$ & $3.86 \pm 2.51$ & $2.40 \pm 0.90$ \\
\hline
\end{tabular}

Tabla II. Promedio de la actividad (min) por especie en condición de perturbación y microhábitat. La significancia de cada comparación se basa en los resultados del MANOVA, en donde ${ }^{*} P<0.05$. Se destacan valores que no son significativos pero se consideran marginales (+; aquellos con un valor de $P=0.0694$ ).

\begin{tabular}{|c|c|c|c|c|}
\hline \multirow[b]{2}{*}{ ESPECIE } & \multicolumn{2}{|c|}{ CONDICIÓN } & \multicolumn{2}{|c|}{ MICROHÁBITAT } \\
\hline & $\begin{array}{c}\text { No } \\
\text { Perturbado }\end{array}$ & Perturbado & Cañada & Ladera \\
\hline Pteronotus parnellii & $8.67 \pm 1.37$ & $8.76 \pm 1.09$ & $8.82 \pm 1.24$ & $8.63 \pm 1.22$ \\
\hline Pteronotus davyi & $10.38 \pm 1.78$ & $9.88 \pm 2.01$ & $12.91 \pm 2.27^{*}$ & $7.85 \pm 1.45$ \\
\hline Pteronotus personatus & $1.50 \pm 0.36$ & $3.6 \pm 0.93$ & $4.09 \pm 0.93^{*}$ & $1.33 \pm 0.46$ \\
\hline Mormoops megalophylla & $4.17 \pm 1.12$ & $8.96 \pm 1.72 *$ & $10.27 \pm 1.73^{*}$ & $3.63 \pm 1.09$ \\
\hline Balantipterix plicata & $7.92 \pm 2.48$ & $11.32 \pm 2.48+$ & $14.59 \pm 3.11^{*}$ & $5.63 \pm 1.59$ \\
\hline Fonotipo VESP & $7.87 \pm 2.00$ & $12.60 \pm 1.99^{*}$ & $13.54 \pm 2.17^{*}$ & $7.63 \pm 1.79$ \\
\hline Fonotipo MOL & $4.70 \pm 1.60$ & $4.48 \pm 1.36$ & $4.27 \pm 1.55$ & $4.85 \pm 1.42$ \\
\hline
\end{tabular}




\section{Discusión}

Al comparar sitios perturbados contra sitios conservados no se encontró un efecto significativo de este factor sobre la actividad de los murciélagos insectívoros aéreos, salvo para M. megalophylla y el fonotipo de los vespertiliónidos, que son más activos en los sitios perturbados, lo cual concuerda con la hipótesis planteada, ya que estas especies son forrajeadoras de borde. El tipo de perturbación que se presenta en la REBIOSH, debido sobre todo al impacto de la ganadería y la extracción de madera (Valenzuela et al., 2010) incrementa las zonas de borde al abrir áreas en la vegetación y reducir el sotobosque.

Para $P$. davyi y $P$. personatus también esperábamos un aumento de la actividad en sitios perturbados, lo cual no sucedió; esto puede deberse a que probablemente para estas especies, los sitios con agua, en este caso los cauces de los arroyos (cañadas), serán sitios más propicios para el forrajeo; A. Guillen (datos no publicados) ha observado a P. personatus forrajeando sobre agua en los mismos sitios donde se hicieron los transectos para medir la actividad relativa de las especies. P. davyi es una de las dos especies más activas en la REBIOSH (la otra es B. plicata, Tablas I y II), y no se registraron diferencias de actividad en relación con la perturbación, por lo cual consideramos que es una de las especies menos afectadas por este factor.

Balantiopterix plicata resultó tener una actividad mayor (aunque marginalmente significativa, Tabla I) en sitios perturbados. Esta especie forrajea alejada de la vegetación o en el borde de la misma, por lo cual este resultado concuerda con lo esperado, ya que al haber perturbación aumentan las superficies de forrajeo.

El efecto del factor temporada sobre los índices de actividad fue solo significativo para B. plicata y el fonotipo MOL. En el caso de B. plicata, que presenta mayor actividad en la temporada seca, se tienen reportados movimientos regionales de los machos en colonias de varias localidades de Morelos y Guerrero: la proporción de machos con relación a las hembras es mayor al inicio del año (temporada seca), durante la época de apareamiento; esta proporción cambia al avanzar el año, siendo baja hacia el final de la temporada de lluvias, lo cual significa una menor cantidad de individuos macho de esta especie en la zona (López-Forment, 1979). Estos movimientos regionales pueden explicar la mayor actividad relativa de la especie en la temporada más seca del año. 
Solo las especies del grupo de los vespertiliónidos siguen un patrón de actividad temporal como el que se esperaba, con más actividad en la época de lluvias; este resultado concuerda con una de nuestras predicciones, aunque esta diferencia solo se presentó en la temporada de lluvias tempranas. Este resultado puede estar influido por un aumento en la cantidad de presas, o por movimientos migratorios o altitudinales de las especies. Por ejemplo, para M. velifera, Villa y Cervantes (2003) reportan que individuos marcados en el Cerro del Ajusco fueron recapturados en el Valle de Cuernavaca durante la primavera y el verano. Para la Sierra de Huautla (ÁvilaTorres Agaton, 2008), se ha encontrado una mayor cantidad de individuos de esta especie en los refugios (minas) durante la época de lluvias tempranas; seguramente debido a movimientos altitudinales, como los registrados por Villa y Cervantes (2003).

Con relación al microhábitat, es notorio que la actividad en las cañadas sea dos o tres veces mayor que en las laderas, con excepción del fonotipo perteneciente a los molósidos y P. parnellii. Estos datos apoyan la idea de que el ensamble en su conjunto prefiere el ambiente de cañada para llevar a cabo sus actividades, sin importar el grado de perturbación de la vegetación circundante.

Las cañadas son sitios generalmente bien conservados en esta zona, debido en parte a las pendientes pronunciadas que las hacen poco aptas para la agricultura e inaccesibles al ganado (Trejo y Dirzo, 2000); aunado a esto y dada la humedad que conservan, algunos elementos florísticos que las componen son perennes, registrándose una mayor productividad de fruta carnosa justo durante la temporada seca del año (De León-Ibarra, 2004). Estos sitios comúnmente mantienen una diversidad de plantas y animales mayor que los sitios adyacentes, ya que ofrecen variedad de hábitats y tipos de alimento, agua, mayor estabilidad climática y refugio en condiciones de mayor estrés ambiental, como las que se presentan en la temporada seca (Bullock y SolísMagallanes, 1990; De León-Ibarra, 2004; Huc, 2005; Meyer et al., 2007; Naiman et al., 1993; Price y Tubman, 2007; Valenzuela y Macdonald, 2002). Particularmente, el agua es indispensable para los murciélagos insectívoros, pues necesitan beberla para hidratarse adecuadamente (Kurta et al., 1989, 1990). Varios trabajos han demostrado la importancia de este tipo de microhábitats para los murciélagos insectívoros en tipos de vegetación muy diferentes (Fukui et al., 2006; Grindal et al., 1999; Rautenbach et al., 1996; Rogers et al., 2006; Seidman y Zabel, 2001).

Las especies que conforman el fonotipo de los molósidos no se vieron afectadas por ninguno de los factores. Estos murciélagos son grandes, con poca maniobrabilidad, vuelo rápido y llamados de ecolocación de baja intensidad y frecuencia constante, por lo que están adaptados al forrajeo en 
áreas abiertas; varios autores mencionan que este tipo de murciélagos, específicamente la familia Molossidae y algunas especies de la familia Vespertilionidae son más activos en lugares abiertos, alejados o libres de vegetación y obstáculos, como los terrenos cultivados o desmontados, y son tolerantes a la perturbación, ya que permanecen en zonas que son deforestadas, a diferencia de otras especies de murciélagos asociados a bosques (Brosset et al., 1996; Fenton et al., 1998; Ochoa, 2000; Patriquin y Barclay, 2003; Rogers et al., 2006). Nuestros resultados concuerdan con la hipótesis planteada, con relación a la ecomorfología de estos murciélagos.

Resulta sumamente interesante encontrar que la actividad de P. parnellii no se ve afectada por ninguno de los factores, principalmente la perturbación, ya que esta especie ha sido catalogada como especialista de hábitats estructuralmente complejos (y por lo tanto bien conservados) de acuerdo a su llamado de ecolocalización y morfología alar (Schnitzler y Kalko, 2001), por lo que inicialmente se consideró que su actividad estaría restringida a sitios con menor perturbación. Una posible explicación a este resultado la encontramos en el trabajo de Pavey et al. (2001), que mencionan que el llamado de ecolocalización de Hipposideros speoris (Andersen) es muy similar al de P. parnellii, con señales de frecuencia constante con un componente final de frecuencia modulada de corta duración. Hipposideros speoris no compensa totalmente por el efecto Doppler, por lo cual no está restringida a forrajear dentro de la vegetación como las especies de la familia Rhinolophidae; esto la hace ser una de las especies más activas y comunes en el mosaico de vegetación de Sigiriya Rock, en Sri Lanka, debido a su capacidad de explotar varios microhábitats de forrajeo. Algo similar debe ocurrir con P. parnellii, en la REBIOSH, ya que de hecho es una de las especies de murciélagos insectívoros más comunes y/o abundantes en las localidades donde se distribuye, de acuerdo a lo reportado por Bateman y Vaughan (1974), Ochoa (2000) y Estrada et al. (2004).

\section{Conclusiones}

En este estudio se registró mayor actividad de murciélagos insectívoros aéreos en las cañadas que en las laderas, lo cual sugiere que, al menos desde el punto de vista de la conservación de este grupo faunístico y del mantenimiento de sus roles ecológicos, la conservación de este microhábitat debería enfatizarse entre las acciones de manejo de esta área natural protegida. 
Los resultados de este trabajo apoyan la importancia de las cañadas para la generalidad de la fauna de las selvas bajas caducifolias y de manera específica, para los murciélagos insectívoros aéreos, y deben considerarse como sitios clave para el monitoreo a largo plazo de este ensamble, en ambientes secos altamente estacionales.

El patrón de actividad estacional que se registró para B. plicata y los vespertiliónidos, parece estar influido por cambios estacionales de tipo reproductivo o movimientos altitudinales determinados por la abundancia de recursos asociada a la estacionalidad.

El poco efecto que tuvieron los factores sobre la actividad de $P$. parnellii, sugiere que es una especie flexible, capaz de utilizar hábitats estructuralmente complejos, pero también sitios con cierto nivel de perturbación; esto se ve reflejado en su abundancia local y en su actividad, medida con los detectores de ultrasonido.

\section{Agradecimientos}

Al CONACyT por el apoyo otorgado mediante la beca con No. de registro: 158263, y por el financiamiento del proyecto de investigación J3490-V “Mastofauna de la Reserva de la Biosfera Sierra de Huautla, Morelos: Diversidad, Patrones Espacio-Temporales y Conservación”, dentro del cual se obtuvieron los datos del presente documento. Al CIByC-UAEM y a pobladores de las comunidades cercanas a los sitios de estudio en la REBIOSH por el apoyo logístico para la realización del trabajo de campo. Los Autores agradecemos al Lic. Gerardo Hernández el diseño gráfico editorial y a la Ms.C. Diana Dorantes la revisión del Idioma Inglés.

\section{Literatura citada}

Adams, J.K. 1989. Pteronotus davyi. Mammalian Species 346: 1-5.

Aldridge, H.D.J. y I.L. Rautenbach. 1987. Morphology, echolocation and resource partitioning in insectivorous bats. Journal of Animal Ecology 56: 763-778.

Avila-Cabadilla L.D., G.A. Sanchez-Azofeifa, K.E. Stoner, M.Y. Alvarez-Añorve, M. Quesada, C. y A. Portillo-Quintero. 2012. Local and Landscape Factors Determining Occurrence of Phyllostomid Bats in Tropical Secondary Forests. PLoS ONE 7(4): e35228. doi:10.1371/journal.pone.0035228

Ávila-Torres Agatón, L. G. 2008. Estimación mediante un sistema audiovisual de las poblaciones de cuatro especies de murciélagos en cinco refugios cavernícolas de la Reserva de la Biosfera Sierra de 
Huautla, Estado de Morelos, México. Tesis de Licenciatura. Facultad de Ciencias Biológicas, Universidad Autónoma del estado de Morelos, Cuernavaca, Morelos.

Bateman, G.C. y T.A. Vaughan. 1974. Nightly activities of mormoopid bats. Journal of Mammalogy 55: 45-65.

Bell, G. 1980. Habitat use and response to patches of prey by desert insectivorous bats. Canadian Journal of Zoology, 58: 1876-1883.

Brigham, R., H. Aldridge, y R. Mackey. 1992. Variation in habitat use y prey selection by Yuma bats, Myotis yumanensis. Journal of Mammalogy 73(3): 640-645.

Brosset, A., P. Charles-Dominique, A. Cockle, J-F. Cosson, y D. Masson. 1996. Bat communities and deforestation in French Guiana. Canadian Journal of Zoology 74: 1974-1982.

Bullock, S.H. y J.A. Solís-Magallanes. 1990. Phenology of canopy trees of a tropical deciduous forest in Mexico. Biotropica 22: 22-35.

Cadena-Salgado M.D. 2003. Efectos de la perturbación y estacionalidad en la comunidad de roedores en una selva baja caducifolia en Morelos, México. Tesis de Licenciatura, Departamento de Química y Biología, Universidad de las Américas Puebla, Santa Catarina Mártir, Puebla. 98 pp.

Ceballos, G. 1995. Vertebrate diversity, ecology and conservation in neotropical dry forest. pp. 195-214. En: S. H. Bullock, H.A. Mooney y E. Medina (eds.). Seasonally dry tropical forest. Cambridge University Press. 455 pp.

De León-Ibarra, M. A. 2004. Fenología y disponibilidad de fruta carnosa en la Sierra de Huautla, Morelos. Tesis de Licenciatura. Facultad de Ciencias Biológicas, Universidad Autónoma del Estado de Morelos. Cuernavaca, Morelos, México.

Estrada, A., C. Jiménez, A. Rivera y E. Fuentes. 2004. General bat activity measured with an ultrasound detector in a fragmented tropical landscape in Los Tuxtlas, Mexico. Animal Biodiversity and Conservation 27(2): 1-9.

Fenton, M.B. 1997. Science and the conservation of bats. Journal of Mammalogy 78(1): 1:14.

Fenton, M.B., D.H.M. Cumming, I.L. Rautenbach, G.S. Cumming, M.S. Cumming, G. Ford, R. D. Taylo, J. Dunlop, M.D. Hovorka, D.S. Johnston, C. V. Portfors, M.C. Kalcounis y Z. Mahlanga. 1998. Bats and the loss of tree canopy in African woodlands. Conservation Biology 12(2): 399-407.

Fukui, D., M. Murakami, S. Nakako y T. Aoi. 2006. Effect of emergent aquatic insects on bat foraging in a riparian forest. Journal of Animal Ecology 75: 1252-1258. 
Glendell, M. y N. Vaughan. 2002. Foraging activity of bats in historic landscape parks in relation to habitat composition and park management. Animal Conservation 5: 309-316.

Grindal, S.D., J.L. Morrissette y R.M. Brigham. 1999. Concentration of bat activity in riparian habitats over an elevation gradient. Canadian Journal of Zoology 77: 972-977.

Gorresen, P.M. y M. R.Willing. 2004. Landscape responses of bats to habitat fragmentation in Atlantic forest of Paraguay. Journal of Mammalogy 85: 688-697.

Hayes, J. P. 1997. Temporal variation in activity of bats and the design of echolocation-monitoring studies. Journal of Mammalogy 78(1): 514-524.

Hayes, J.P. 2000. Assumptions and practical considerations in the design and interpretation of echolocationmonitoring studies. Acta Chiropterologica 2: 225-236.

Huc, T. 2005. Estudio de la diversidad de artrópodos del suelo y evaluación de su uso como bioindicadores en la Reserva de la Biosfera Sierra de Huautla. Tesis de Licenciatura. Escuela de Ciencias, Universidad de Las Américas-Puebla. Puebla, México.

Ibáñez C., A. Guillén, J. Juste B., y J. L. Pérez-Jordá.1999. Echolocation calls of Pteronotus davyi (Chiroptera: Mormoopidae) from Panama. Journal of Mammalogy 80(3): 924-928.

Ibáñez C., J. Juste, R. López-Wilchis, L. Albuja V. y A. Núñez-Garduño. 2002. Echolocation of three species of sac-winged bats (Balantiopterix). Journal of Mammalogy 83(4): 1049-1057.

Jong, J. 1994. Choice of foraging sites in a bat community. En: Distribution patterns and habitat use by bats in relation to landscape heterogeneity, and consequences for conservation. Tesis doctoral. Department of Wildlife Ecology. Swedish University of Agricultural Sciences

Jong, J. y I. Ahlén. 1991. Factors affecting the distribution patterns of bats in Uppland, central Sweden. Holarctic Ecology 14: 92-96.

Kalko, E.K.V., C.O. Handley Jr. y D. Handley. 1996. Organization, diversity, and long-term dynamics of a neotropical bat community. Pp. 503-533. En: M. L. Cody y J. A. Smallwood (eds). Long-term studies of vertebrate communities. Academic Press. 597 pp.

Korine, C. y B. Pinshow. 2004. Guild structure, foraging space use, and distribution in a community of insectivorous bats in the Negev Desert. Journal of Zoology 262: 187-196.

Kurta, A., G. P. Bell, K. A. Nagy y T.H. Kunz. 1989. Water balance of free-ranging little brown bats (Myotis lucifugus) during pregnancy and lactation. Canadian Journal of Zoology 67(10): 2468- 
2472.

Kurta, A., T. H. Kunz, y K. A. Nagy. 1990. Energetics and water flux of free-ranging big brown bats (Eptesicus fuscus) during pregnancy and lactation. Journal of Mammalogy, 71(1), 59-65.

López-Forment, C.W. 1979. Algunos aspectos ecológicos del murciélago Balantiopterix plicata Peters, 1987 (Chiroptera: Emballonuridae) en México. Anales del Instituto de Biología 50, Ser. Zoología: 673-699.

Macias, S. E., C. Mora y A. García. 2006. Acoustic identification of mormoopid bats a survey during the evening exodus. Journal of Mammalogy 87(2): 324-330.

MacSwiney, M.C., P. Vilchis, F. M. Clarke y P. A. Racey. 2007. The importance of cenotes in conserving bat assemblages in the Yucatan, Mexico. Biological Conservation 136: 499-509. doi:10.1016/j. biocon.2006.12.021

Meyer, C. F., C. J. Schwarz y J. Fahr. 2004. Activity patterns and habitat preferences of insectivorous bats in a West African forest-savanna mosaic. Journal of Tropical Ecology 20: 397-407.

Meyer, J. L., D. Strayer, B. Wallace, S. L. Eggert, G. S. Helfman y N. E. Leonard. 2007. The contribution of headwater streams to biodiversity in river networks. Journal of the American Water Resources Association 43(1): 86-103.

Mickleburgh, S.P., A. M. Hutson y P. A. Racey. 2002. A review of the global conservation status of bats. Oryx 36(1): 18:34.

Miller, B.W. 2001. A method for determining activity of free flying bats using a new activity index for acoustic monitoring. Acta Chiropterologica 3(1): 93-105.

Naiman, R. J., H. Décamps y M. Pollock. 1993. The role of riparian corridors un maintaining regional biodiversity. Ecological Applications 3(2): 209-212.

Neuweiler, G. 1989. Foraging ecology and audition in echolocating bats. Trends in Ecology and Evolution 4: 160-166.

Novick A. 1962. Orientation in neotropical bats. I. Natalidae and Emballonuridae. Journal of Mammalogy 43(4): 449-455.

O’Farrell, M.J. y B. W. Miller. 1997. A new examination of echolocation calls of some neotropical bats (Emballonuridae and Mormoopidae. Journal of Mammalogy 78(3): 954-963.

O’Farrell M. J. y W.L. Gannon. 1999. A comparison of acoustic versus capture techniques for the inventory of bats. Journal of Mammalogy 80: 24-30. 
O’Farrell, M., B.W. Miller, y W. L. Gannon. 1999. Qualitative identificacion of free-flying bats using the Anabat detector. Journal of Mammalogy 80(1): 11-23.

Ochoa, J. 2000. Efectos de la extracción de maderas sobre la diversidad de mamíferos pequeños en bosque de tierras bajas de la Guayana Venezolana. Biotropica 32(1): 146-164.

Orozco-Lugo, L., A. Guillén-Servent, D. Valenzuela-Galván, y H. T. Arita. 2013. Descripción de los pulsos de ecolocalización de once especies de murciélagos insectívoros aéreos de una selva baja caducifolia en Morelos, México. THERYA 4(1): 33-46. DOI: 10.12933/therya-13-103

Orozco-Lugo, C. L., D. Valenzuela-Galván, A. Guillén-Servent, A. Lavalle-Sánchez y A. J. RhodesEspinoza. 2014. First record of four bat species for the state of Morelos and new bat records for the Sierra de Huautla Biosphere Reserve, Mexico. Revista Mexicana de Biodiversidad 85: 38-47. DOI: $10.7550 / \mathrm{rmb} .40012$

Parsons, S. y G. Jones. 2000. Acoustic identification of twelve species of echolocating bats by discriminant function analysis and artificial neural networks. The Journal of Experimental Biology 203: 26412656.

Patriquin, K. J. y R. M. R. Barclay. 2003. Foraging by bats in clared, thinned and unharvested boreal forest. Journal of Applied Ecology 40: 646-657.

Pavey, C.R., J-E. Grunwald y G. Neuweiler. 2001. Foraging habitat and echolocation behaviour of Schneider's leafnosed bat, Hipposideros speoris, in a vegetation mosaic in Sri Lanka. Behavioral Ecology and Sociobiology 50: 209-218.

Pineda, E., C. Moreno, F. Escobar, y G. Halffter. 2005. Frog, bat, and dung beetle diversity in the cloud forest and coffee agroecosystems of Veracruz, México. Conservation Biology 19(2): 400-410.

Price, P. y W. Tubman. 2007. Structure and characteristics of riparian lands. Pp. 1-12. En: Lovett, S. y P. Price (eds.) Principles for Riparian Lands Management. Land \& Water Australia. Canberra, Australia. 174 pp.

Ratcliffe, J.M., H.M. ter Hofstede, R. Avila-Flores, M. B. Fenton, G.F. McCracken, S. Biscardi, J. Blasko, E. Gillam, J. Orprecio y G. Spanjer. 2004. Conespecific influence call design in the Brazilian free-tailed bat, Tadarida brasiliensis. Canadian Journal of Zoology 82: 966-971.

Rautenbach, I. L., M.B. Fenton y M.J. Whiting. 1996. Bats in riverine forests and woodlands: a latitudinal transect in southern Africa. Canadian Journal of Zoology 74: 312-322.

Reis, N. dos R. y M. F. Muller. 1995. Bat diversity of forest and open areas in subtropical region of South 
Brazil. Ecología Austral 5(31): 31-36.

Rhodes, A. J. 2005. Estructura y diversidad espacio temporal de la comunidad de murciélagos con relación a gradientes ambientales en la Reserva de la Biosfera Sierra de Huautla. Tesis Licenciatura. Escuela de Ciencias, Universidad de Las Américas-Puebla. Puebla, México.

Rogers, D.S., M.C. Belk.M.W.González y B.L. Coleman. 2006. Patterns of habitat use by bats along a riparian corridor in northern Utah. The Southwestern Naturalist 51(1): 52-58.

Rydell J., H.T. Arita, M. Santos y J. Granados. 2002. Acoustic identification of insectivorous bats (order Chiroptera) of Yucatán, México. Journal of Zoology 257: 27-36.

Sánchez-Azofeifa, G.A., M. Quesada, J. P. Rodríguez, J. M. Nassar, K. E. Stoner, A. Castillo, T. Garvin, E. L. Zent, J. C. Calvo-Alvarado, M. E. R. Kalacska, L. Fajardo, J. A. Gamon y P. Cuevas-Reyes. 2005. Research Priorities for Neotropical Dry Forests. Biotropica 37(4): 477-485. DOI: 10.1046/j.0950-091x.2001.00153.x-i1

Sánchez-Hernández, C., y M. L. Romero. 1995. Mastofauna silvestre del área de Reserva Sierra de Huautla (con énfasis en la región noreste). Centro de Investigaciones Biológicas, Universidad Autónoma del Estado de Morelos. Cuernavaca, México. 146 pp.

Schnitzler, H. U. y E. Kalko. 1998. How echolocating bats search and find food. pp. 183-196. En: Kunz, T. H. y P. A. Racey (eds.). Bat Biology and Conservation. The Smithsonian Institution. Washington, USA. 365 pp.

Schnitzler, H. U. y E. Kalko. 2001. Echolocation by insect-eating bats. BioScience 51(7): 557-569.

Schnitzler, H. U., C. F. Moss y A. Denzinger. 2003. From spatial orientation to food acquisition in echolocating bats. Trends in Ecology and Evolution 18(8): 386-394.

Seidman. V. M. y C. J. Zabel. 2001. Bat activity intermittent streams in northwestern California. Journal of Mammalogy 82(3): 738-744.

Stoner, K. E., M. Quesada, V. Rosas-Guerrero y J. A. Lobo. 2002. Effects of forest fragmentation on the Colima Long-nosed bat (Musonycteris harrisoni) foraging in tropical dry forest of Jalisco, Mexico. Biotropica 34(3): 462-467.

Trejo, I. y R. Dirzo. 2000. Deforestation of seasonally dry tropical forest: a national and local analysis in Mexico. Biological Conservation 94: 133-142.

Valenzuela, D. y D.W. Macdonald. 2002. Home range use by white-nosed coatis: limited water and a test of the Resource Dispersion Hypothesis. Journal of Zoology 258: 247-256. 
Valenzuela, D., R. Ramírez y O. Dorado. 2010. Ficha Técnica 29 Sierra de Huautla. Pp. 477-481. En: G. Ceballos, E. Espinoza, J. Bezaury y A. García (eds.). Diversidad, amenazas y regiones prioritarias para la conservación de las selvas secas del Pacífico de México. CONABIO y FCE. México, D.F., México. 594 pp.

Villa R., B. y F. A. Cervantes. 2003. Los mamíferos de México. Instituto de Biología UNAM y Grupo Editorial Iberoamerica. 140 pp.

Webala, P.w., N. O. Oguge y A. Bekele. 2004. Bat species diversity and distribution in the three vegetation communities of Meru National Park, Kenia. African Journal of Ecology 42: 171-179.

Wickramasinghe, L. P., S.Harris, G. Jones y N. Vaughan. 2003. Bat activity and species richness in organic and conventional farms: impact of agricultural intensification. Journal of Applied Ecology 40: 984-993.

Zubaid, A. 1993. A comparison of the bat fauna between a primary and fragmented secondary forest in Peninsular Malaysia. Mammalia 57(2): 201-206.

\section{CITA:}

Orozco- Lugo C.L, A. Guillen-Servent, D. Valenzuela-Gálvan, H. T. Arita y N. A. Mariano-Bonigo. 2017. Variación espacio-temporal en la actividad relativa de murciélagos insectívoros aéreos en la Reserva de la Biosfera Sierra de Huautla, Morelos, México. Áreas Naturales Protegidas Scripta, 2017. Vol. 3 (1): 35-57. https:// doi.org/10.18242/anpscripta.2017.03.03.01.0002

Sometido: 23 de Julio de 2016

Revisado: 17 de Octubre de 2016

Aceptado: 26 de Marzo de 2017

Editor asociado: Dr. Luis Ignacio Iñiguez Dávalos

Idioma Inglés Abstract: Ms.C. Diana Dorantes

Diseño gráfico editorial: Lic. Gerardo Hernández 


\section{Anexo 1}

Listado de especies y asignación de grupos funcionales (GF) de murciélagos insectívoros aéreos reportados para la Reserva de la Biosfera Sierra de Huautla. Abreviaturas. IA= Insectívoro aéreo, IAC= insectívoro aéreo de espacios cerrados (dentro de la vegetación), IAA= insectívoro aéreo de espacios abiertos (alejado de la vegetación), IAB= insectívoro aéreo de borde (cerca o en huecos en la vegetación), IS= insectívoro de sustrato, * No hay datos suficientes para definir un gremio de manera concluyente

\begin{tabular}{|c|c|c|c|}
\hline Familia & Especie & $\begin{array}{l}\text { GF, Schnitzeler y Kalko } \\
\text { (2001), Schnitzeler } \text { et al. } \\
\text { (2003) }\end{array}$ & $\begin{array}{c}\text { GF, Guillen (observación personal) } \\
\text { para individuos de la zona de } \\
\text { estudio }\end{array}$ \\
\hline Emballonuridae & Balantiopterix plicata & IAA & IAA e IAB \\
\hline \multirow[t]{6}{*}{ Molossidae } & Eumops underwoodi & IAA & IAA \\
\hline & Eumops ferox & IAA & \\
\hline & Molossus sinaloe & IAA & IAA \\
\hline & Nyctinomops femorosaccus & IAA & \\
\hline & Nyctinomops macrotis & IAA & \\
\hline & Tadarida brasiliensis & IAA & IAA \\
\hline \multirow[t]{4}{*}{ Mormoopidae } & Mormoops megalophylla & $\mathrm{IAB}$ & $\begin{array}{c}\text { IA vuelo rasante sobre vegetación y } \\
\text { sobre agua }\end{array}$ \\
\hline & Pteronotus parnellii & IAC & IAC \\
\hline & Pteronotus davyi & IAB & IAB \\
\hline & Pteronotus personatus & IAB & IA vuelo rasante sobre agua \\
\hline Natalidae & Natalus mexicanus & & IAC \\
\hline \multirow[t]{12}{*}{ Vespertilionidae } & Eptesicus fuscus & IAB & IAB \\
\hline & Lasiurus blossevilli & IAA & \\
\hline & Lasiurus cinereus & IAA & IAB \\
\hline & Lasiurus intermedius & IAA & \\
\hline & Myotis californicus & IAB & \\
\hline & Myotis fortidens & IAB & \\
\hline & Myotis yumanensis & IAB & IA asociado a cuerpos de agua* \\
\hline & Myotis velifer & IAB & $\begin{array}{c}\mathrm{IAB}, \text { ocasionalmente forrajea sobre } \\
\text { agua }\end{array}$ \\
\hline & Parastrellus hesperus & IAB & \\
\hline & Rhogeessa alleni & & IA \\
\hline & Rhogeessa gracilis & & $\mathrm{IAC}^{*}$ \\
\hline & Rhogeessa parvula & & $\mathrm{IAC}^{*}$ \\
\hline
\end{tabular}

